\title{
A high result in wavelet watermarking using singular value decomposition
}

\author{
Wellia Shinta Sari ${ }^{\star 1}$, Christy Atika Sari ${ }^{2}$ \\ Universitas Dian Nuswantoro ${ }^{1,2}$
}

\author{
Article Info \\ Keywords: \\ Watermarking, Image, Discrete Wavelet \\ Transform (DWT), Singular Value \\ Decomposition (SVD)
}

\section{Article history:}

Received 4 December 2018

Revised 28 February 2019

Accepted 21 June 2019

Published 30 July 2019

\section{Cite:}

Sari, W., \& Sari, C. (2019). A High Result in Wavelet Watermarking Using Singular Value Decomposition. Kinetik: Game Technology, Information System, Computer Network,

Computing, Electronics, and Control, 4(3).

doi:http://dx.doi.org/10.22219/kinetik.v4i3.729

* Corresponding author.

Wellia Shinta Sari

E-mail address:

Wellie.shinta.sari@dsn.dinus.ac.id

\begin{abstract}
Internet that has developed into a good distribution tool for digital data, causing a large increase in digital data sharing, especially in the form of digital images, and causing problems that need attention. One of them is about copyright protection. Watermarking is one technique that aims to protect digital image copyright. In this study, watermarking was carried out using the Discrete Wavelet Transform (DWT) and Singular Value Decomposition (SVD) algorithms in digital images with different file extensions. Transformation of 2 levels of DWT and SVD on the host image and SVD transformation on the watermarked image that inserted in the LL2 sub-band of the host image. Watermarking with the proposed method produces good image quality with PSNR values exceeding $40 \mathrm{~dB}$, SSIM reaching 0.99 and NCC reaching 1 . This shows a robust and imperceptible watermarking image.
\end{abstract}

\section{Introduction}

The internet has become a good means of distribution for digital data which has led to a large increase in the sharing of digital data such as images, audio and video via the internet. So that problems arise that need to be considered to protect digital data, one of which is about copyright protection. Watermarking has the potential to meet the needs of the problem because the workings of watermarking are by inserting information that shows purpose, ownership, or other information on digital media without affecting its quality [1] and will not disappear during normal use, so that digital data owners can claim the data is his property by extracting the information that has been inserted before. In general, watermarking on digital images can be done in the spatial domain or frequency domain (transformation). Spatial domain watermarking, although it is simple and has a smaller level of time complexity but is not strong enough to withstand attacks and watermarks are easily detected by other parties [2]. The frequency domain watermarking is stronger than the spatial domain because of the insertion of the watermark into the changing frequency coefficient of the changing image [3]. Frequency domain-based watermarking is more widely used because of its robustness and imperceptible which are the main requirements for watermarking techniques [4]. The most commonly used frequencybased watermarking is Discrete Cosine Transform (DCT), Discrete Fourier Transform (DFT), and Discrete Wavelet Transform (DWT). DCT and DWT watermarking techniques are implemented very effectively in digital watermarking image schemes [5].

Until now, many methods have been proposed to insert a watermark. As research has been conducted by [4] which also combines the concepts of DWT and SVD in watermarking. In the study, the algorithm used was focused on improving the quality of the watermark image and the resistance of the watermark to be inserted. The proposed technique is able to achieve the imperceptibility, robustness and security of watermarks. Both quantitative and qualitative results show that the watermark is invisible and robust against attaks.

Whereas in research [6], a comparison of the watermarking technique between IWT (Integer Wavelet Transform)SVD and DWT-SVD was carried out. Comparison is done by including variations in the value of the watermark ( $\alpha$ ) visibility factor. The quality of watermarking is measured using PSNR and NCC. With variations in the value of $\alpha$ from 0.1 to 1 , it is known that the smaller the value of $\alpha$, the greater the value of PSNR but vice versa with the value of NCC. The experimental results show that the quality of the watermark image and extracted watermark depends only on the scaling factor. This can also be seen from the simulation results that for the same scaling factor, IWT-SVD-based watermarking shows better results than the DWT-SVD as far as the PSNR value is concerned. 
In this paper, the watermarking technique used is a combination of Discrete Wavelet Transform (DWT) level 2 and Singular Value Decomposition (SVD) to protect the copyright of a digital image with a different image file extension. By combining the two techniques, it is expected that the results of watermarking will be more robust and imperceptible.

\section{Research Method}

\subsection{Discrete Wavelet Transform (DWT)}

DWT is a mathematical tool for splitting images without changing the contents of the information in the signal. This transformation provides a spatial description and frequency of a digital image [7]. Wavelet transforms describe digital images in four non-overlapping multi resolution sub-bands, namely (Figure 1):

1. Low-low (LL) sub band

2. Low-high (LH) sub band

3. High-low $(\mathrm{HL})$ sub band

4. High-high $(\mathrm{HH})$ sub band

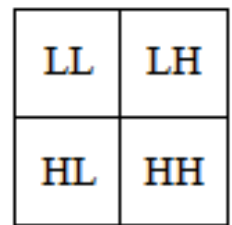

Figure 1. 1L-DWT Decomposition [8]

Wavelet-based watermarking methods are used to obtain resilience which also keeps the two types of information transformed, namely frequency and spatial information. The bandwidth of the determination coefficient (LL) gives a greater DWT coefficient at each decomposition level and other bands $(\mathrm{HH}, \mathrm{HL}$ and $\mathrm{LH})$ which give a smaller coefficient value [9]. Therefore, watermark insertion in other sub-bands $(\mathrm{HH}, \mathrm{HL}$ and $\mathrm{LH})$ can reduce image quality. Whereas, inserting a watermark in the LL sub band can improve image resistance [7].

In reconstruction analysis, the DWT inverse is carried out by the same structure as the corresponding synthesis sub-image, which consists of inverse quadrant coefficients and detail coefficients. This feature makes watermarks stronger than spatial methods for various distortions. The application of DWT is efficient for two important reasons, namely [10]:

1. DWT has an irregular distribution of inverse transformation values in the pyramidal wavelet domain.

2. DWT has low linear complexity that requires low computational costs $O(n)$ when compared to the computational costs consumed by Fourier and cosine transformations $\mathrm{O}(\mathrm{n} \log (\mathrm{n}))$.

3. DWT can use wavelet filters for image transformation. There are many types of filters that can be used in transformation. Filters that are commonly used for watermarking techniques are [9]: Haar Wavelet Filter, Daubechies Orthogonal Filters, Daubechies Bi-Orthogonal Filters, Symlet wavelets, Morlet wavelets, Coiflets wavelets, Meyer wavelets.

The DWT transformation process is done by dividing the digital image into a $2 \times 2$ non-overlap block [8] as show in Equation 1.

$$
\left[\begin{array}{cc}
I_{m, n} & I_{m, n+1} \\
I_{m+1, n} & I_{m+1, n+1}
\end{array}\right] \text { or }\left[\begin{array}{ll}
a & b \\
c & d
\end{array}\right]
$$

Where $I_{m, n}$ is the intensity value of the pixel digital image in the position of row $\mathrm{m}$ and column $\mathrm{n}$. The following is an example of DWT transformation on digital images with filters that will be used in this study, Haar filter.

\subsection{Singular Value Decomposition (SVD)}

Singular Value Decomposition (SVD) is a popular mathematical technique that provides tools for matrix analysis [11]. SVD is a numerical analysis of linear algebra which is an important topic among mathematicians [8]. SVD was first introduced by Beltrami and Jordan in 1870 for a square matrix and then Eckart and Young in 1936 extended to a rectangular matrix [11]. SVD has special features that can be done on a real-world matrix $(m, n)$. For example, there is a matrix $A$ with rows of $m$ matrices and column length matrices $n$. Then, $A$ can be factored into 3 (three) matrix $U, S$ and $\mathrm{V}$ with the following composition [8] as show in Equation 2.

$$
A=U_{m m} S_{m n} V^{T}{ }_{n n}
$$

(c) 2019 The Authors. Published by Universitas Muhammadiyah Malang

This is an open access article under the CC BY SA license. (https://creativecommons.org/licenses/by-sa/4.0/) 


\section{Where:}

$\mathrm{U}$ matrix is an orthogonal matrix with the order $\mathrm{m} \times \mathrm{n}$ which is $U^{T} U=I$.

$\mathrm{V}$ matrix is an orthogonal matrix with the order $\mathrm{m} \times \mathrm{n}$ which is $V^{T} V=I$.

The column of $\mathrm{U}$ is an orthonormal eigenvector of $A A^{T}$

The column of $\mathrm{V}$ is an orthonormal eigenvector of $A^{T} A$

$\mathrm{S}$ is a diagonal matrix containing the eigenvalue roots of $\mathrm{U}$ and $\mathrm{V}$ arranged in sequence from the largest to the smallest value.

Singular image values have been widely used in pattern recognition and image analysis, SVD has two advantages, namely [12]:

1. Geometric invariant in orthogonal, transformation, rotation and zoom.

2. Good stability and anti-noise.

There are several important characteristics of SVD, namely [12]:

1. The singular value of the image is the main intrinsic characteristic of the image, but not the visual characteristics, the largest single value coefficient contains most energy features.

2. Stability is an interesting one from the singular value of the image, the singular value of the image will not change if the image is applied to a small disturbance.

3. Singular values are invariants of images such as rotation, translation, zoom, transpose and other geometric distortions.

\section{Proposed Scheme}

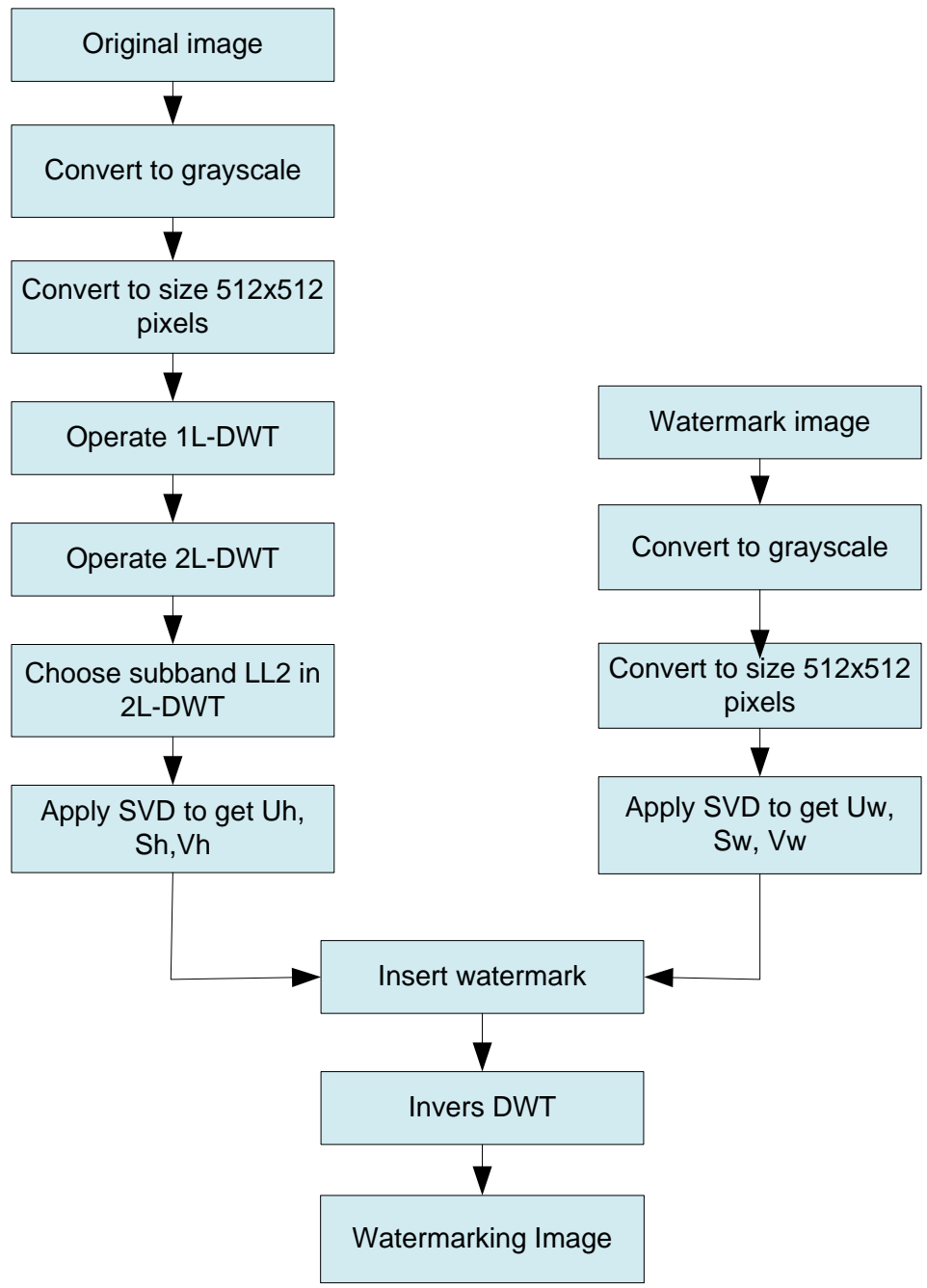

Figure 2. Embedding Scheme Using 2L-DWT and SVD 


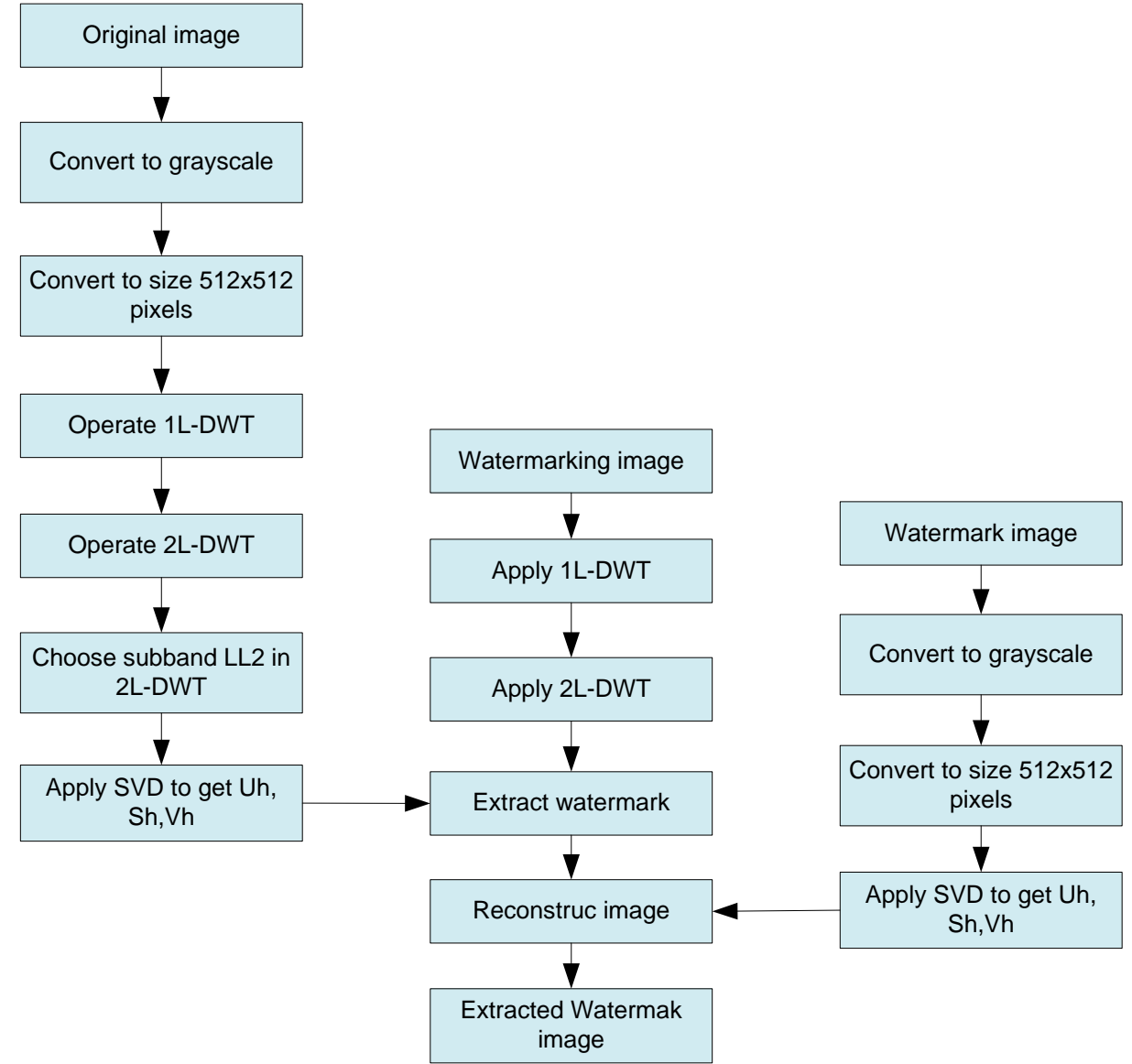

Figure 3. Extracting Scheme Using 2L-DWT and SVD

The following is a way of working from combining watermarking techniques which consist of a watermark insertion process into the original image and a watermark image extraction process to recover the watermark that has been inserted in the original image as show in Figure 2 and Figure 3.

\section{Results and Discussion}

The method proposed in this study is image watermarking by combining the techniques of 2 levels of Digital Wavelet Transform (DWT) and Singular Value Decomposition (SVD). Before the embedding process is carried out, digital images are inputted as original images (hosts) and watermark images in the form of color images but in the process the images will be converted into grayscale images with the grayscale process as in [13]. Then, the host image will be resized to $512 \times 512$ pixels.

The image used in this study amounted to 18 images including 5 host images with each ending in JPEG, BMP and TIFF image files and 1 watermark image with each ending JPG, BMP and TIFF image files as show in Figure 4. Whereas, Figure 5 represent watermark in this proposed paper.

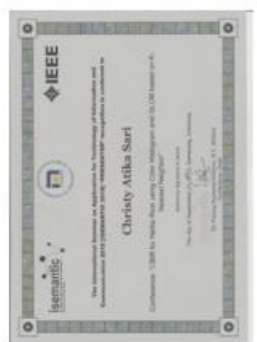

S1

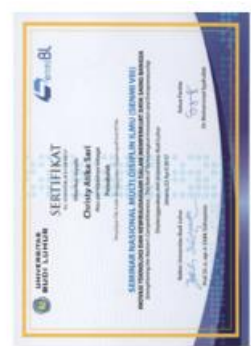

S2

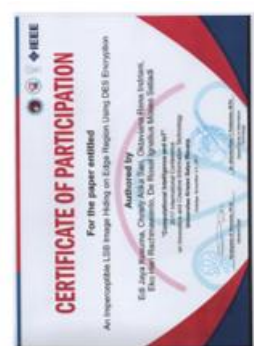

S3

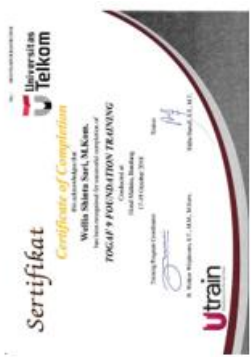

S4

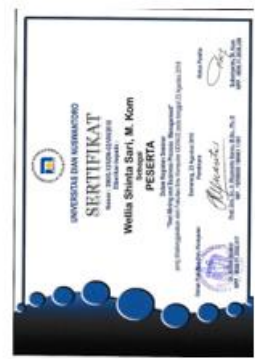

S5

Figure 4. Sample of Host Images (512x512 pixels)

(c) 2019 The Authors. Published by Universitas Muhammadiyah Malang

This is an open access article under the CC BY SA license. (https://creativecommons.org/licenses/by-sa/4.0/) 


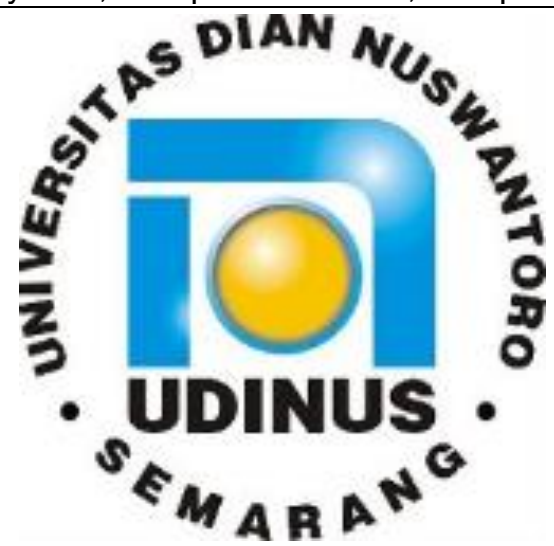

Figure 5. Message Image (128x128 pixels)

Here, we have tested our results such as MSE, PSNR, SSIM, and NCC. PSNR (Peak Signal to Noise Ratio) and MSE (Mean Square Error) are image quality measuring instruments that are measured based on statistical errors or differences in intensity between images before being processed with images after processing [8]. PSNR is a metric used to evaluate the difference or imperceptibility between the original image and the watermarked image that measures the image quality and is described in decibels $(\mathrm{dB})$ [14]. For 8-bit images or PSNR values commonly found are between 30 to $50 \mathrm{~dB}$ [15],[16],[17]. The MSE is defined as the average square of the difference in the pixel value of the two images. MSE serves to assess how well a method performs image reconstruction or restoration relative to its original image [8]. The values of MSE and PSNR are obtained by the Equation 3 and Equation 4.

$$
\begin{gathered}
P S N R=10 \log _{10}\left(\frac{255 \times 255}{M S E}\right) \\
M S E=\frac{1}{N M} \sum_{i=1}^{N} \sum_{j=1}^{M}[(G w(i, j)-G(i, j))]^{2}
\end{gathered}
$$

SSIM is an image quality measuring instrument whose measurement is based on the characteristics of HVS (Human Visual System) [8]. SSIM is a metric that is used to detect how far the difference in images is distorted when compared to the original image in terms of structural information [4]. The SSIM value is obtained by the follow in Equation 5.

$$
\operatorname{SSIM}(x, y)=\frac{\left(2 \mu_{x} \mu_{y}+C_{1}\right)\left(\sigma_{x y}+C_{2}\right)}{\left(\mu_{x}^{2}+\mu_{y}^{2}+C_{1}\right)\left(\sigma_{x}^{2}+\sigma_{y}^{2}+C_{2}\right)}
$$

The resulting SSIM index is a decimal value between 0 and 1, value 1 can only be achieved in the case of two identical data sets.

While, NCC is an image quality measuring instrument whose measurement is based on the similarity of two images, namely the image before processing and the image after processing. NCC is a correlation function that aims to measure the level of closeness or similarity between two digital images [8]. NCC measures the level of image similarity using the Equation 6.

$$
N C C=\frac{\sum_{X=1}^{N} \sum_{y=1}^{M}\left[f_{1}(x, y) \cdot f_{2}(x, y)\right]}{\sqrt{\left(\sum_{X=1}^{N} \sum_{y=1}^{M}\left[f_{1}(x, y)\right]^{2}\right)\left(\sum_{X=1}^{N} \sum_{y=1}^{M}\left[f_{2}(x, y)\right]^{2}\right)}}
$$

NCCs have a range of values from 0 to 1 . The NCC values that indicate value 1 indicate identical images. Table 1 below is the result of a trial of the host image that we use.

As presented Table 1, very good visual results were obtained. The image is pressed by just cutting the center with the aim of inserting the message in the center of the image. After the watermarking process, image quality testing is done by looking for the values of MSE, PSNR, SSIM and NCC to determine image quality after the watermarking process is performed. 

Table 1. Example Visual Result of Embedding Image Without Attacks

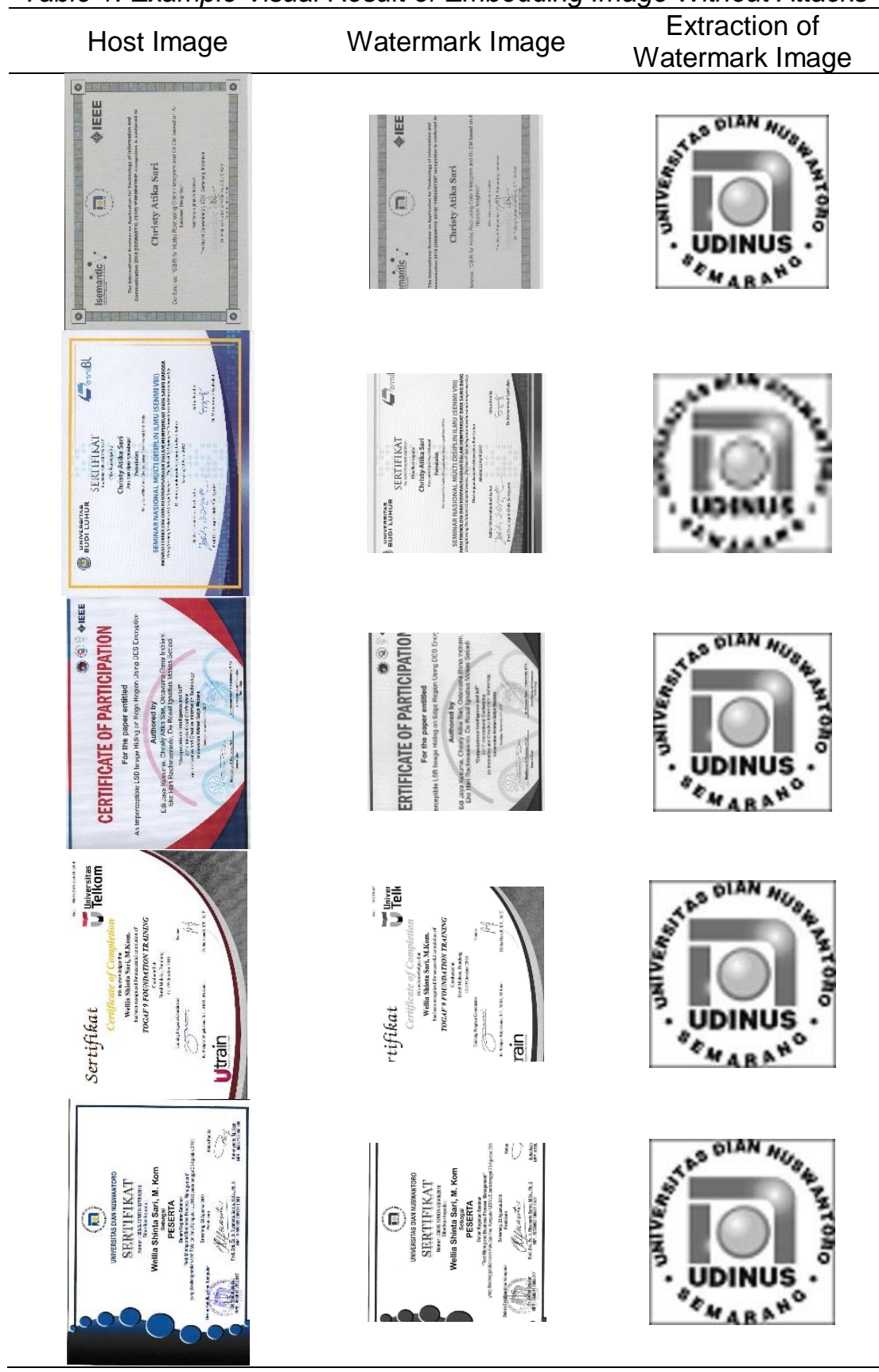

Table 2. A Result of MSE, PSNR, SSIM and NC

\begin{tabular}{ccccc}
\hline Image & MSE & PSNR & SSIM & NCC \\
\hline JPEG \& JPG & 2.03285 & 45.04975 & 0.999094 & 1 \\
BMP \& JPG & 2.03285 & 45.04975 & 0.999094 & 1 \\
TIFF \& JPG & 2.03285 & 45.04975 & 0.999094 & 1 \\
JPEG \& BMP & 2.03285 & 45.04974 & 0.999098 & 1 \\
BMP \& BMP & 2.03285 & 45.04974 & 0.999098 & 1 \\
TIFF \& BMP & 2.03285 & 45.04974 & 0.999098 & 1 \\
JPEG \& TIFF & 2.02897 & 45.05804 & 0.999098 & 1 \\
BMP \& TIFF & 2.02897 & 45.05804 & 0.999098 & 1 \\
TIFF \& TIFF & 2.02897 & 45.05804 & 0.999098 & 1 \\
Average & 2.030522 & 45.05472 & 0.999098 & 1 \\
\hline
\end{tabular}

(c) 2019 The Authors. Published by Universitas Muhammadiyah Malang

This is an open access article under the CC BY SA license. (https://creativecommons.org/licenses/by-sa/4.0/) 
Based on Table 2 above, it is known that the host image inserted in JPG format watermark image gets the same value of MSE, PSNR and SSIM even though the host image has a different file format. Likewise, the host image is inserted with BMP and TIFF watermark images. From the results of the PSNR and SSIM values obtained, it proves that the watermarking algorithm used in this study results in digital image watermarking that has an impedance or has a degree of similarity between the host image and high watermarked image. From all experiments conducted, the average PSNR value is 45.05251 and the average SSIM value is 0.99909 which is close to its maximum value, namely 1 . From all experiments, the extracted watermark image also gets the maximum NC value of 1 which shows the similarity and image quality well.

In Table 3, a comparison of image quality by watermarking and JPEG image format and JPG image watermark image using the Level 1 DWT algorithm, Level 2 DWT, Level 1 DWT-SVD algorithm and the algorithm proposed in this study with the value of $\alpha=0.025$. 2L-DWT has better imperceptibility than 1L-DWT [4], and our proposed method proved it. This study achieve high performance based on 2L-DWT in [4], we also prove it by PSNR value.

Table 3. A Comparison of PSNR Value

\begin{tabular}{|c|c|c|c|c|}
\hline \multirow{2}{*}{$\begin{array}{l}\text { Name of } \\
\text { image }\end{array}$} & \multicolumn{4}{|c|}{ PSNR (dB) } \\
\hline & 1DWT & 2DWT & 1LDWT-SVD & 2LDWT-SVD \\
\hline $\mathrm{S} 1$ & 33.00848 & 33.01289 & 39.02778 & 45.04975 \\
\hline S2 & 33.00848 & 33.01289 & 39.02778 & 45.04975 \\
\hline S3 & 33.00848 & 33.01289 & 39.02778 & 45.04975 \\
\hline S4 & 33.00848 & 33.01289 & 39.02778 & 45.04975 \\
\hline S5 & 33.00848 & 33.01289 & 39.02778 & 45.04975 \\
\hline Average & 33.00848 & 33.01289 & 39.02778 & 45.04975 \\
\hline \multicolumn{5}{|c|}{ Table 4. A Comparison of NCC Value } \\
\hline \multirow{2}{*}{$\begin{array}{l}\text { Name of } \\
\text { image }\end{array}$} & \multicolumn{4}{|c|}{ PSNR (dB) } \\
\hline & 1DWT & 2DWT & 1LDWT-SVD & 2LDWT-SVD \\
\hline S1 & 0.99961 & 0.99871 & 1 & 1 \\
\hline S2 & 0.99978 & 0.99910 & 1 & 1 \\
\hline S3 & 0.99959 & 0.99867 & 1 & 1 \\
\hline S4 & 0.99926 & 0.99756 & 1 & 1 \\
\hline S5 & 0.99755 & 0.99485 & 1 & 1 \\
\hline Average & 0.999142 & 0.997778 & 1 & 1 \\
\hline
\end{tabular}

Based on the exposure of Table 4, it can be seen that the 2 level DWT-SVD watermarking algorithm has the highest PSNR value compared to other watermarking algorithms. Whereas for the NCC value, it does not have a significant difference even though the NCC watermarking value of 2 DWT levels is smaller than 1 DWT level. From the table above shows the results of the average NCC value of the extracted watermark image that does not have a significant difference.

\section{Conclusion}

The application of watermarking by using a combination of Discrete Wavelet Transform (DWT) and Singular Value Decomposition (SVD) methods has been proven to produce high image with imperceptibility and robustness. This is shown from the PSNR value in the trial with the use of the JPG, BMP and TIFF file formats generated 45.05804 $\mathrm{dB}$ where the message image used is in the TIFF format. On the other hand, the SSIM obtained is 0.999098 , and the NCC in all images produces a perfect value of 1 .

In experiments with JPEG image format and JPG image watermark produced the highest PSNR of $45.04975 \mathrm{~dB}$ obtained by 2LDWT-SVD. This proves that the performance of 2LDWT-SVD is better than 1LDWT, 2LDWT, and 1LDWT-SVD. Based on the trial it is known that the NCC value obtained also gets a value of 1 in all images. Can claim digital images from watermarking by extracting watermark images from watermarked images. With an NCC value of 1 , it can prove ownership rights of the digital image. Image file extensions that are used as host images or watermark images do not affect the quality of watermarking images. This can be proven by the average value of MSE, PSNR, SSIM and NCC in each experiment that did not have a significant difference.

\section{References}

[1] P. N. Andono and T. Sutojo, Konsep Pengolahan Citra Digital, Revisi ed., Semarang: CV. Andi Offset, 2015.

[2] V. Yadav, D. P. Singh and J. Kaur, "DWT-SVD Based Digital Image Watermarking Using GA," International Journal of Science, Engineering and Technology Research (IJSETR), Vol. 4, No. 7, July 2015.

[3] P. Singh and R. S. Chadha, "A Survey of Digital Watermarking Techniques, Applications and Attacks," International Journal of Engineering and Innovative Technology (IJEIT), Vol. 2, No. 9, Pp. 165-175, Maret 2013.

Cite: Sari, W., \& Sari, C. (2019). A High Result in Wavelet Watermarking Using Singular Value Decomposition. Kinetik: Game Technology, Information System, Computer Network, Computing, Electronics, and Control, 4(3). doi:http://dx.doi.org/10.22219/kinetik.v4i3.729 
[4] J. Kaur, N. Singh and C. Jain, "An Improved Image Watermarking Technique Implementing 2-DWT and SVD," India, 2016. https://doi.org/10.1109/RTEICT.2016.7808156

[5] B. P. D. a. K. M. S. Y. Shantikumar Singh, "A Review of Different Techniques on Digital Image Watermarking Scheme," International Journal of Engineering Research, Vol. 2, No. 3, Pp. 193-199, Juli 2013.

[6] P. Gupta and G. Parmar, "Image Watermarking using IWT-SVD and its Comparative Analysis with DWT-SVD," in International Conference on Computer, Communications and Electronics (Comptelix), Jaipur, India, 2017. https://doi.org/10.1109/COMPTELIX.2017.8004026

[7] P. Gupta and G. Parmar, "Image Watermarking Using IWT-SVD and its Comparative Analysis with DWT-SVD," Jaipur, 2107. https://doi.org/10.1109/COMPTELIX.2017.8004026

[8] P. N. Andono and M. T. Sutojo, Pengolahan Citra Digital, Semarang: ANDI.

[9] R. Choudhary and G. Parmar, "A Robust Image Watermarking Technique Using 2-level Discrete Wavelet Transform (DWT)," in IEEE 2nd International Conference on Communication, Control and Intelligent Systems (CCIS), Mathura, India, 2016. https://doi.org/10.1109/CCIntelS.2016.7878213

[10]H. Tao, L. Chongmin, J. M. Zain and A. N. Abdalla, "Robust Image Watermarking Theories and Techniques: A Review," Vol. 12, No. 1, 2014.

[11]D. K. Thind and S. Jindal, "A Semi Blind DWT-SVD Video Watermarking," in International Conference on Information and Communication Technologies (ICICT 2014), 2015. https://doi.org/10.1016/j.procs.2015.02.104

[12]Z. Nana, "Watermarking Algorithm of Spatial Domain Image Based On SVD," Shanghai, 2016. https://doi.org/10.1109/ICALIP.2016.7846588

[13] https://www.mathworks.com/help/wavelet/ref/dwt2.html

[14]P. Shah, T. Meenpal, A. Sharma, V. Gupta and A. Kotecha, "A DWT-SVD Based Digital Watermarking Technique for Copyright Protection," in International Conference on Electrical, Electronics, Signals, Communication and Optimization (EESCO), 2015.

[15] K. Ji, J. Lin, H. Li, A. Wang and T. Tang, "A DCT And SVD Based Watermarking Technique to Identify Tag," 2015.

[16]T. T. Takore, P. R. Kumar and G. L. Devi, "A Modified Blind Image Watermarking Scheme Based on DWT, DCT and SVD Domain Using GA to Optimize Robustness," in International Conference on Electrical, Electronics, and Optimization Techniques (ICEEOT), Chennai, 2016. https://doi.org/10.1109/ICEEOT.2016.7755190

[17][Online]. Available: https://www.mathworks.com/help/images/ref/ssim.html. [Accessed 30 January 2018].

(c) 2019 The Authors. Published by Universitas Muhammadiyah Malang

This is an open access article under the CC BY SA license. (https://creativecommons.org/licenses/by-sa/4.0/) 